\title{
Cationisation of alginate fiber to improve the dyeing properties using natural dyes from rhubarb: a new approach
}

\author{
Md. Kawshar Ahamed ${ }^{1}$, Mohammad Raza Miah ${ }^{1 *}$, Most. Munera khatun ${ }^{2}$, Md. Hasan-Al \\ Mamun ${ }^{1}$, Cui $\mathrm{Li}^{1}$ \\ ${ }^{1}$ College of Chemistry and Chemical Engineering, Wuhan Textile University, Hubei, China \\ ${ }^{2}$ Department of Environmental Chemistry, China University of Geosciences, Hubei, China \\ *Corresponding author: razawtu2015@gmail.com
}

\begin{abstract}
In this investigation dyeing of alginate fiber was carried out after cationic treatment of EPTAC (2, 3-Epoxypropyl Trimethyl Ammonium Chloride). Optimum dyeing process and parameter show good dyeing performance when alginate fiber dyed with rhubarb dye. In addition to cationic treatment is taken into account for alginate fiber to improve fastness, color strength, breaking strength. Later in dyeing for fixation ferrous sulfate, copper sulphate was considered as mordents. Moreover using pre-mordanting methods conveyed the dyeing of alginate fiber with the stave of metallic mordant and without metallic salt mordents. The evaluation of each color dyed material was done through following two terms for instance CIELAB $\left(\mathrm{L}^{*}, \mathrm{a}^{*}\right.$, and $\left.\mathrm{b}^{*}\right)$ and $\mathrm{K} / \mathrm{S}$ values. According to AATCC test methods color fastness to washing of the dyed fiber was determined whereas according to the ASTM D3822M standard the breaking strength of alginate fiber was estimated and tested. When dyeing was carried out on alginate fiber through considering optimum parameter like $80{ }^{\circ} \mathrm{C}$ for $90 \mathrm{~min}, \mathrm{M}$ : $\mathrm{L} 1: 40$ and at $\mathrm{pH} 7$ which showed optimum results. In addition to mostly very good wash fastness was obtained while there was no fading of the color, whereas the moderate level of color fastness to crocking was achieved. Later after dyeing the evaluations of SEM of the cationized alginate fiber and FTIR of powder from the rhubarb dye were observed. The results suggested that the surface of cationized alginate fiber was smoother than the raw alginate fiber.
\end{abstract}

Key words Alginate fiber, rhubarb natural dye, color strength \&fastness, SEM, Eco-friendly

\section{Introduction}

With the consistent for new substances, always greater efficient, extra dependable and of more performance, one would not clearly don't forget seaweeds as an ability elegance (Wang et al., 2015). However, now not most effective do they play an essential function within the ecological machine (algae are the idea of all food chains inside the sea offering as much as half of the earth's oxygen), however a few have additionally been used for hundreds of years as food, as manure or as a supply of chemical compounds. Alginates, a form of brown seaweed, have been accrued in Scotland for the reason that 18th century for the treatment of blisters and lacerations. 
From the 1960's, seaweeds have been farmed and nowadays, alginates locate vast applications in both the fabric, food and the pharmaceutical industries (Badwan, Abumalooh, Sallam, Abukalaf, \& Jawan, 1985; Burrows \& Welch, 1983; Dey, Khan, \& Chowdhury, 2011; King, 1983; Luo, Lode, \& Gelinsky, 2013; Yimin, 2003; ZHAN, ZHU, ZHANG, \& GUO, 2006; Zhu, Zhang, Sui, $\&$ Wang, 2009). The profit increased from the usage of these herbal polymers derives specially from their capacity to shape a hydrogel with most divalent cations, inclusive of $\mathrm{Ca} 2+$ ions. Moreover, their excessive absorbency and inherent haemo compatibility lead them to of precise attention within the making of wound dressings. Thirty years in the past, it become found that wounds heal higher in a wet surroundings additionally, on touch with blood, calcium alginate rapidly releases the calcium ions in trade for sodium ions, stimulating each platelet activation and entire blood coagulation. Previous research task became predominantly proposed to gain a larger perception into ion change ion binding within alginates (Hong-bin, 2005). The second factors of hobby become a way to improve traits which includes electricity and swelling of those biomaterials. This was accomplished following the course of polymer blends which include alginate/pectin (a polysaccharide particularly ample in end result) and alginate/carboxy methyl cellulose (essential constituent of the mobile wall of plant life). The third factors of polysaccharide structures were considered in this painting: pure alginates, alginate/pectin blends and alginate/carboxy methyl cellulose blends. The have a look at of every system changed into completed following the identical pattern.

\subsection{Literature review}

\subsubsection{Alginate fiber}

Alginates are hydrophilic polysaccharides found in brown seaweed, comprising of as much as forty wt. \% of dry depend (the seaweed consists of other ionic polymers including fucoidan). They occur within the intercellular matrix as a mixed sodium-magnesium-calcium-strontium gel, the relative proportions of those ions being determined via an ion-change equilibrium response with sea water (Morris, Rees, Thom, \& Boyd, 1978). Their important feature is skeletal, giving both electricity and flexibility to the algal tissue (Skjåk-Bræk, 1992). Those polymers have found tremendous industrial use due to their capability to form a gel with divalent cations which includes $\mathrm{Ca} 2+$. A method for the extraction of alginates becomes patented initially with the aid of Stanford in 1881. But the first products from the Stanford method have been very crude (they contained high tiers of nitrogen) (Siviello, 2016). Kelco business enterprise (San Diego) appeared to be the primary to produce and to commercialize pure sodium alginates, within the 1920 's. These days, about 15000 tons dry weight of alginates are processed yearly from four hundred 000 tons of seaweed (Gacesa, 1988) maximum of the alginates are extracted from just three of the 265 reported species of the marine brown algae. Macrocytic is the most commonplace one and is harvested off the west coast of America. Further Luminaries and Ascophyllum are obtained from Northern Europe. The choice on which seaweed to method commercially is based totally upon the price of the uncooked cloth and its alginate content. Every other important criterion is the type of alginate contained inside the seaweed, since the 
structure of the alginate determines its overall performance (see I.A.2). At the same time as seaweed is the principle supply of alginates, several microbial assets have additionally been observed, like Azotobacter vinelandii and Pseudomonas aeruginosa. Unlike seaweed alginates, bacterial alginates are acetylated. Despite the fact that they're no longer at gift broadly used, bacterial alginates might end up a possible opportunity as a marine pollutant has produced instability within the long-time period seaweed alginate supply (Ott \& Day, 1995).

\subsubsection{Manufacturing process}

For commercial utility alginate fibers want a few special purification because of its inner morphology have a few inherent residences that needs special care to make alginate fibers suitable for software level the economic production system of alginate fiber changed into represented (Siviello, 2016).

In phrases of the producing process, calcium alginate fiber can be made thru one of the maximum simple spinning processes. The spinning solution can be made through dissolving sodium alginate powder in water and, after degassing to get rid of the bubbles within the answer, a focused sodium alginate solution may be extruded thru best spinneret holes right into a calcium chloride tub, whereby sodium alginate is precipitated out in filament form as a calcium alginate fiber (the latter being insoluble in water). The as-made fibers can then be stretched, washed and dried to provide calcium alginate fibers. Figure 2 suggests schematically a small wet-spinning manufacturing line. It have to be talked about that answers of sodium alginate can react with many di- and trivalent cations to form gels; hence it's far viable to apply a ramification of metal ions to precipitate sodium alginate answer at some stage in the wet-spinning technique. Within the production of alginate fibers, calcium has observed greatest recognition as the divalent ion for gel formation, especially because its salts are cheap, without difficulty to be had and nontoxic. Zinc chloride has also been used for the manufacturing of zinc alginate fibers (Qin, Agboh, Wang, \& Gilding, 1996).

Figure 3 shows the egg-container model for alginate gel formation (Grant, Morris, Rees, Smith, \& Thom, 1973) all through the extrusion process where sodium alginate is extruded right into a calcium chloride tub, the buckled chain of guluronic acid gadgets acts as a two-dimensional analogue of a corrugated egg-container with interstices wherein the calcium ions may $\%$ and be coordinated, and even as calcium ions assist to maintain the alginate molecules together, their polymeric nature and their aggregation bind the calcium ion greater firmly, resulting in a company gel structure.

Previous study revealed that the shape of the guluronic acid block gives distances among carboxyl and hydroxyl organizations which permit a high degree of coordination of the calcium ion. At some point of the production of calcium alginate fibers, calcium ions from the coagulation bathtub diffuse into the fiber to form a swollen fibrous gel. (Thomas, Gilson, \& Ahmed, 1995) studied the diffusion of calcium ions at some point of the wet-spinning technique. Their consequences showed that the gelling time of calcium alginate fiber numerous linearly 
with alginate attention, extended markedly with fiber radius and reduced with increasing calcium attention. Its miles thrilling that the gelling time is independent of the guluronic acid and mannuronic acid contents.

\section{Materials and methods}

\subsection{Materials}

Natural dyes rhubarb with different degrees of staining on the alginate fiber, were selected in this study. They were purchased from the Changzhou Mel sheng Biomaterials Co. Ltd, China. Acetic acid and Sodium acetate which were used without any purification to adjust the dyeing bath $\mathrm{pH}$ were supplied by Sino pharm Chemical Reagent Co. Ltd, China. For surface modification of alginate fiber cationic agent EPTAC (2, 3-Epoxypropyl Trimethyl Ammonium Chloride), ferrous sulphate, copper sulphate, sodium hydroxide was used and sodium hydroxide supplied from Shanghai ZhuoRui Chemical Co. Ltd, China. All these chemicals were analytical grade. Lab dyeing machine was used to dye the alginate fiber which was purchased from Guangdong province. A vacuum woven dryer was used which was purchased from Yu Ming Instrument co. ltd. of Shanghai.

\subsection{Methods}

\subsubsection{Cationic treatment of alginate fiber}

Cationic treatment of alginate fiber was done by using $3 \mathrm{~g} / \mathrm{l}$ cationic agent EPTAC (2, 3Epoxypropyl Trimethyl Ammonium Chloride) at temperature $60^{\circ} \mathrm{C}$ for $30 \mathrm{~min}$. Later alginate fiber was dried at vacuum woven dryer.

\subsubsection{Mordanting method}

In case of dyeing of natural dyes, different types of mordant are used at pre-mordanting method. Here, ferrous sulphate, copper sulphate mordant, was used during the dyeing process to find out the best result for alginate fiber with rhubarb dyes. $40 \mathrm{~g} / 1$ ferrous sulphate, copper sulphate solution was made and further it was used in together with the dyes solution. When mordant was used together with rhubarb dyes, it increased the affinity of the dyes to the alginate fiber. During dyeing process, mordant were applied in different conical flax with the dyes solution to find out the best dyeing results. Every conical flax will be marked with different numbering sticker to identify the beakers after the dyeing process (Vankar, Shanker, \& Verma, 2007).

\subsubsection{Dyeing of alginate fiber with rhubarb dye}

Dyeing Recipe

Alginate fiber $=2-4 \mathrm{gm}$

$\mathrm{pH}=4-8$

Temperature $=40^{\circ}$ to $90^{\circ} \mathrm{C}$

Time $=40-90 \mathrm{~min}$

Rhubarb dye $=5 \%$ (o.w.f) 
$\mathrm{M}: \mathrm{L}=1: 10-1: 40$

After preparing the alginate fiber, dyes solution and mordant dyeing process was started. At first dyeing solution of rhubarb dye (5\% o.w.f) were taken in different 5 conical flax. Then $40 \mathrm{~g} / \mathrm{l}$ mordant was given in different flax by using a pipette. One flax was without mordant for checking the different dyeing result with other samples. Finally, alginate fiber was placed in the conical flax solution and shaken for sometimes for a proper mixing of dye solution with the fiber (LIU \& GAO, 2010). The laboratory dyeing machine temperature was set at different temperature from $40^{\circ}$ to $90^{\circ} \mathrm{C}$ for dyeing process. It takes some time to reach the required temperature and when the temperature reached at required temperature, the conical flax given to the machine and run the machine for different duration from 40-120 min at various concentrations from 1:20-1:45 for different $\mathrm{pH}$ 5-12. The machines was shaken by setting a proper rpm in the machine. After different time duration the conical flax was taken out from the machine.

\subsubsection{After treatment}

After dyeing process, the dyed samples were taken out from the flax and kept in a bowl. Hot wash treatment was done for 2-3 minutes to remove the unfixed dyes from the surface of the dyed alginate fiber. After hot wash, soaping was done. $5 \mathrm{gm}$. $/ 1$ detergent was used to make the soap solution. $1000 \mathrm{ml}$ water was taken in a beaker and $5 \mathrm{gm}$. detergent was weighted. Then mixed together in the beaker and soap solution was ready for the soaping treatment. The soaping process was done in a 1:30 ratio and $120 \mathrm{ml}$ soap solution was taken in every conical flax. Every conical flax again set into the dyeing machine and run the machine at $60^{\circ} \mathrm{C}$ for 10 minutes. After soaping, the samples were washed by using cold water. After cold wash, every sample were properly rinsed. After rinsed, the samples were given to the vacuum dryer. The samples were dried in oven dryer at $80^{\circ} \mathrm{C}$ for about 20 minutes

\subsubsection{Testing and analysis}

\subsubsection{FTIR Analysis}

The FTIR spectrum of rhubarb dye particle was recorded by using FTIR spectrophotometer (Brukar vertex 100, Germany). Fourier Transform Infrared Spectroscopy uses infrared light to scan test samples and observe chemical properties like organic, inorganic and polymeric materials. FTIR analysis accomplished by Attenuated Total Reflectance (ATR), Nujol and thin film. Solid samples are usually prepared by shaving some material off of the sample that is thin enough to obtain a good spectrum. The spectrum was recorded in transmittance mode by using ATR sampling technique (Skoda, Astier, Pajonk, \& Primet, 1994). The KBr plate containing dye particles were prepared and the spectrums were recorded over 4000-400 cm-1 wavelength. The average reading of 40 scans were recorded in $1 \mathrm{~cm}$ interval. 


\subsubsection{Scanning Electron Microscope Infrared Spectroscopy of Rhubarb dye (SEM)}

The surface morphology of Rhubarb dye particles was investigated by using scanning electron microscope (phoenix 5000, shanghai). Gold Nano particle sputtering was applied on dye particles to ensure the opacity caused by electron beams. The sample was scanned by using $5 \mathrm{kv}$ electron intensity and samples were examined with magnifications ranging from 1000 to 5000X times. Scanning Electron Microscope gives the electronic image which reflects the morphology of the sample surface. Scanning Electron Microscope was performed at high magnifications, generates high-resolution images and precisely measures very small features and objects.

\subsubsection{Evaluation of color strength}

Color strength of the Rhubarb dyed alginate fiber was evaluated by using the spectrophotometer machine. A data color machine of $110 \mathrm{TM}$ was purchased from Shanghai used to measure the color strength of the dyed samples. At first the cotton dyed sample was placed in the data color and different values of the dyed sample was found like lightness, value, hues and color strength of the dyed sample was measured by $(\mathrm{K} / \mathrm{S})$ value. The CIELAB color space.

The color difference is expressed by the following equation 1

$\mathrm{DE} *=(\mathrm{DL} *) 2+(\mathrm{Da} *) 2 \mathrm{Db} *)$

Here $\mathrm{DE}^{*}$ is the CIELAB color difference between batch and standard. Here DL*, $\mathrm{Da}^{*}, \mathrm{Db}^{*}$ and hence $\mathrm{DE}^{*}$ are in commensurate units. $\mathrm{DL}^{*}$ denotes the difference between lightness (where $\left.\mathrm{L}^{*}=100\right)$ and darkness (where $\left.\mathrm{L}^{*}=0\right), \mathrm{Da}^{*}$ the difference between green $\left(\mathrm{a}^{*}\right)$ and red $\left(+\mathrm{a}^{*}\right)$ and $\mathrm{Db}^{*}$ the difference between yellow $\left(+\mathrm{b}^{*}\right)$ and blue $\left(-\mathrm{b}^{*}\right)$ (fig). All measured samples showed a maximum absorption.

\subsubsection{Color fastness to wash test}

ISO-105-C06 standard washing fastness test method was applied in case of dyed alginate fiber. A washing fastness machine was used for wash fastness test which was purchased from Shandong province. At first, $4 \mathrm{gm}$. Detergent, $1 \mathrm{gm}$. Na-par borate and $1000 \mathrm{ml}$ water were mixed together in a beaker to make the washing solution. The alginate dyed fiber was cut according to the size $10 \mathrm{~cm} \times 4 \mathrm{~cm}$. Multi-fiber fabric was also cut according to the same size \& attached with the dyed sample. Every sample were marked with different markings to find out the washing fastness results. Then $50 \mathrm{ml}$ washing solution was taken in different conical flax. The attached dyed sample with multi fiber fabric was placed in the conical flax \& 25 steel balls were given in every conical flax. After this, the conical flax was given to the washing machine from Shangdong Company at $60^{\circ} \mathrm{C}$ for 30 minutes. After 30 minutes, washed samples were taken out from the machine $\&$ cold wash was done. After washing rinsing was done $\&$ given to the dryer. After drying the samples were checked with the help of grey scale.

\subsubsection{Breaking strength of rhubarb dyed alginate fiber}

After cat ionization treatment and pre-mordanting process breaking strength of rhubarb dyed alginate fiber was investigated according to ASTM D3822M method (Properties, 1996). 


\section{Results and discussions}

\subsection{FTIR analysis}

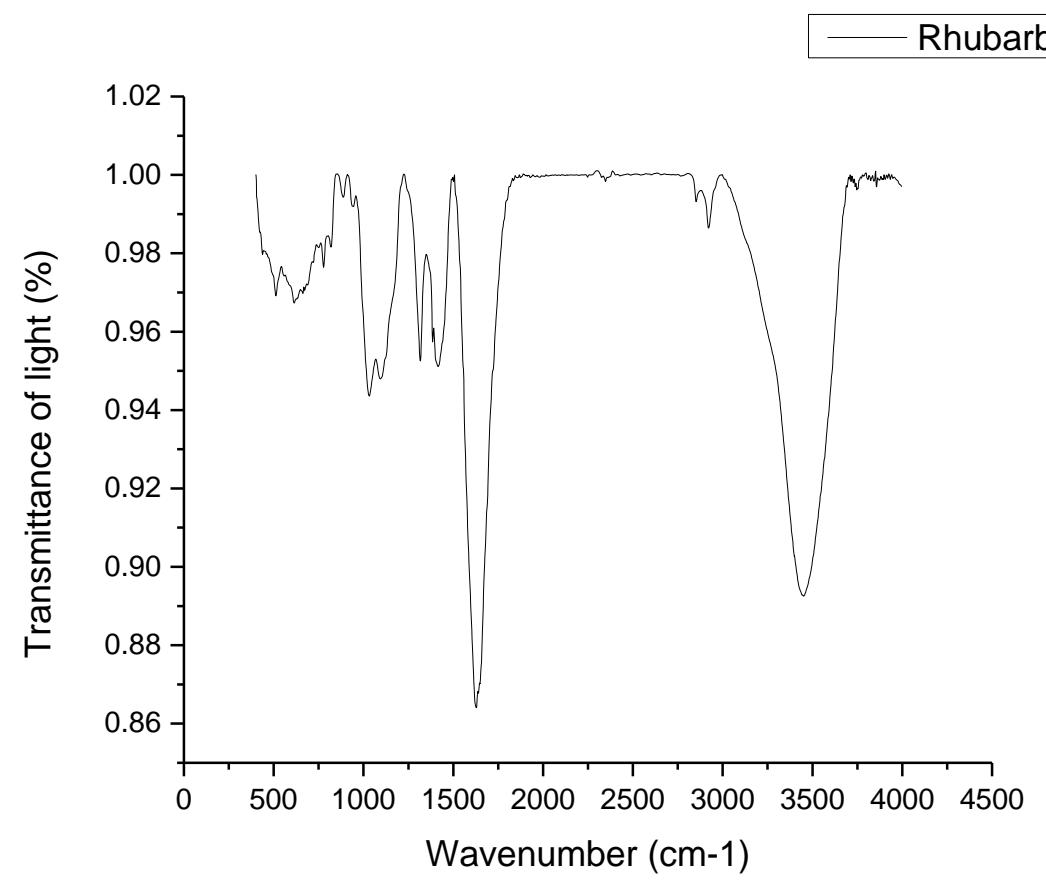

Figure 1: Fourier Transform Infrared Spectroscopy of rhubarb dye

In addition to Fourier Transform, Infrared Spectroscopy was used to analyze and identify polymer, dye functional group with respect to different wavelengths that generate the transmittance of light spectra which exhibit the unique chemical bonds and the molecular structure of rhubarb dye at Figure 1. Furthermore, transmittance of light spectrum demonstrates zenith and peak that schematically represent components in higher concentration. Additionally peaks on the spectrum indicate functional groups for instance alkanes, ketones, acid chlorides, alcohol, carboxylic acids, esters, ethers group. However due to various bonds and different functional groups dye molecule absorb different infrared radiation wavelengths. Moreover, functional groups of rhubarb dye were investigated from the graph according to the standard characteristics of IR absorptions. However within the wavelength range 515-690 cm-1 there is a medium stretch in the graph which indicates that in this position alkyl halide group $(\mathrm{C}-\mathrm{Br})$ is present. Moreover in the wavelength of $850-550 \mathrm{~cm}-1$ there is a medium stretch in the graph which represents that in this position alkyl halide group $(\mathrm{C}-\mathrm{Cl})$ is exist. In addition to the wavelength range of 950-910 cm-1 there is a medium O-H bend in the graph which shepherd that in this position carboxylic acids are extant. In the wavelength range $1000-1320 \mathrm{~cm}-1$ there is a strong stretch which usher that alcohols, carboxylic acids, esters, ethers group (C-O) are present there. 
According to the graph, $1150-1300 \mathrm{~cm}-1$ there is a medium wag which escort that alkyl halide group $(-\mathrm{CH} 2 \mathrm{X})$ is alive in dye molecule. Moreover, aromatic groups $(\mathrm{C}-\mathrm{C})$ were identified by a medium stretch in the wavelength 1500-1400 cm-1. In the case of wavelength, 1580-1650 cm-1 medium bend of the figure indicates that 10 amine group $(\mathrm{N}-\mathrm{N})$ is present there.

In the wavelength range $3200-3500 \mathrm{~cm}-1$ there is a strong broad stretch which shows that those alcohols and phenols groups (O-H group) are present there. In the wavelength range 3610-3640 cm-1 there is a strong sharp stretch which indicates that there are free hydroxyl alcohols and phenols group (O-H group) is present in sappon wood. Thus, different functional groups of sappon wood were investigated with respect to standard characteristics of the infrared spectroscopy. In a nutshell hydroxyl group and alkyl halide are a dominating group among the various functional groups by analyzing the morphology of rhubarb dye.

\subsection{Factors affecting dyeing properties}

\subsubsection{Temperature}

Alginate tries to dye at low temperature move again a few years. It's been diagnosed for decades that fiber harm as a result of dyeing is reduced markedly while alginate is dyed at boiling temperature. On this research work emphasis on saving power through dyeing of cationised alginate fiber with mordant and dyeing of alginate fiber with mordant lowering the fundamental issues resulting from excessive temperature dyeing of alginate fiber. Previous study revealed that alginate dyeing is the one of the primary problems of sometimes uneven dyeing. If the alginate cuticle is damaged via localized chemical attack or by using abrasion. At higher temperature, the extra polar the dye is greater strongly its miles absorbed by the damaged fibers. But this absorption is very choppy and at better temperature the unevenness is even greater seen than at lower temperature. But the depth of colour achieved is extra at better temperatures compared to lower temperatures. At excessive temperature, alginate fiber has a tendency to become worse in energy and turns into yellow and offers harsh sense/handle. The influence of temperature on alginate fiber during dyeing with rhubarb dye was presented at 
Figure 2. Color strength of alginate fiber increased from $40^{\circ}$ to $80^{\circ} \mathrm{C}$ temperature. When temperature was above $80^{\circ} \mathrm{C}$ color strength of was decreased due the physical and chemical nature of alginate fiber and dye molecule. Cationic treatment of alginate fiber improves the color strength as well as exhaustion and fixation of dye molecule.

\subsubsection{Time}

The influence of time on alginate fiber during dyeing with rhubarb dye was presented at

Figure 3. It was seen from that, the color strength increased with increasing time. The absorption of the dye molecules commenced on the much less ordered surfaces of the fibers after which proceeded underneath favorable situations into the interlinking place among elementary crystallites, penetrating into them from each ends. Simplest water-swollen intermicellar areas and regions of decrease order of the fibers have been reachable to massive acid dyes molecules. The molecular chains in amorphous vicinity exhibited disorderly packing and could circulate effortlessly to provide a big free extent, permitting dye molecules to diffuse effortlessly into and out of the chains. Equilibrium between adsorption and desorption was accordingly displaced. As the dye attention extended, the probability of touch among the dye molecules and fibers' surface multiplied. But, increasing the attention improved the aggregation of the dye molecules inside the dye tub, and that turned into prejudicial for dye molecules to penetrate into the fibers. And an excess of dye concentration would reason the waste of the dyes and water pollution. 


\subsection{3 pH}

$\mathrm{pH}$ play vital role in the field of wet processing specially in dyeing different type of fiber and fabric. In textile dyeing $\mathrm{pH}$ keep the fiber properties by maintaining the exhaustion between fiber and dye molecule. The influence of $\mathrm{pH}$ on alginate fiber during dyeing with rhubarb dye was presented at Figure 4. Color strength of alginate fiber increased from 3 to $7 \mathrm{pH}$ due to the nature and structure of alginate fiber.Alginate fiber alter its insoluble stage into soluble stage when goes into water. Hydrogen bond and van der Waals forces facilitates the dyeing process by making reaction between fiber and rhubarb dye .However alginate fiber make ionic bond with rhubarb dye that contain anion. Uneven dyeing was occurred at lower $\mathrm{pH}$ and higher $\mathrm{pH}$ increased the depth of shade. However higher $\mathrm{pH}$ reduced the rate of dyeing. The positive impact of this higher $\mathrm{pH}$ is that the improving the level of dyeing.

\subsubsection{Dye concentration}

The influence of dye concentration on alginate fiber during dyeing with rhubarb dye was presented at

Figure 5. It was seen from that, the color strength increased with increasing dye concentration. The absorption of the dye molecules commenced on the much less ordered surfaces of the fibers after which proceeded underneath favorable situations into the interlinking place among elementary crystallites, penetrating into them from each ends. Simplest water-swollen intermicellar areas and regions of decrease order of the fibers have been reachable to massive acid dyes molecules. The molecular chains in amorphous vicinity exhibited disorderly packing and could circulate effortlessly to provide a big free extent, permitting dye molecules to diffuse effortlessly into and out of the chains. Equilibrium between adsorption and desorption was accordingly displaced. As the dye attention extended, the probability of touch among the dye molecules and fibers' surface multiplied. But, increasing the attention improved the aggregation of the dye molecules inside the dye tub, and that turned into prejudicial for dye molecules to penetrate into the fibers. And an excess of dye concentration would reason the waste of the dyes and water pollution. 

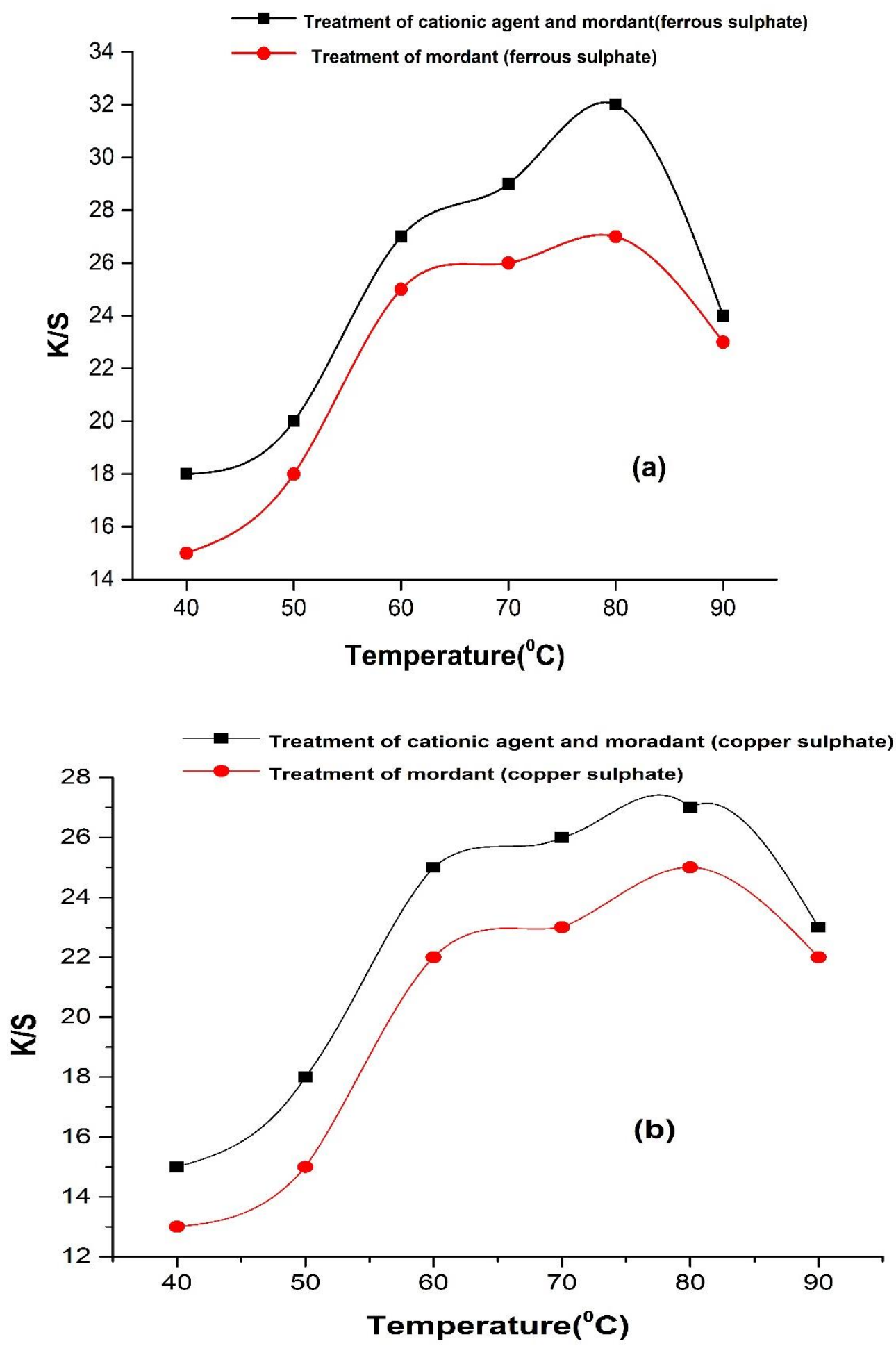

Figure 2: Effect of dyeing temperature on the color strength of alginate fiber with (a) cationic agent and ferrous sulphate treated, (b) cationic agent and copper sulphate treated during premordanting process(dyeing condition: time $90 \mathrm{~min}, \mathrm{M}: \mathrm{L}=1: 40, \mathrm{pH}=7$ ) 

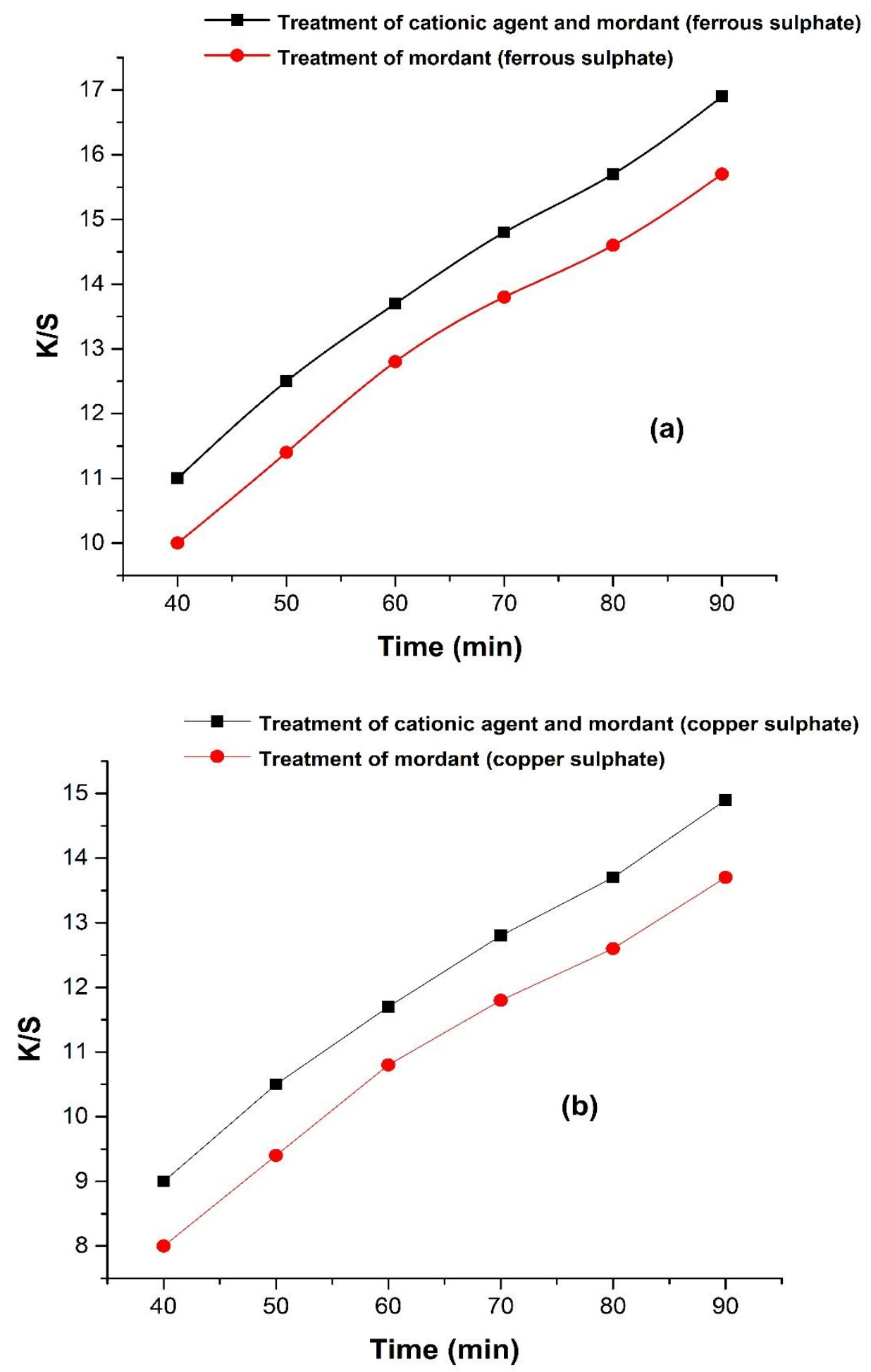

Figure 3: Effect of dyeing time on the color strength of alginate fiber with (a) cationic agent and ferrous sulphate treated, (b) cationic agent and copper sulphate treated during pre-mordanting process(dyeing condition: temperature $80^{\circ} \mathrm{C}, \mathrm{M}: \mathrm{L}=1: 40, \mathrm{pH}=7$ ). 

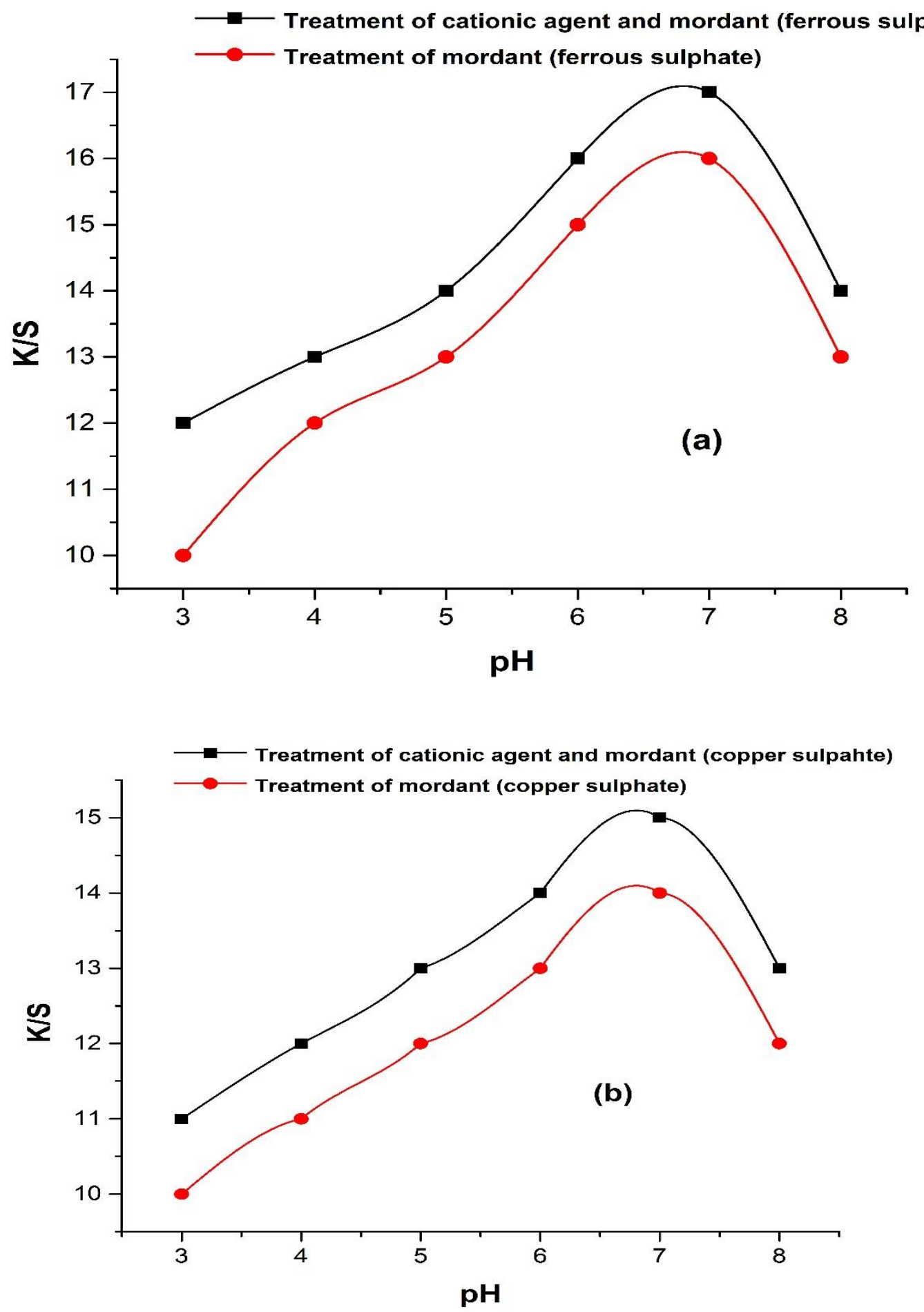

Figure 4: Effect of dyeing $\mathrm{pH}$ on the color strength of alginate fiber with (a) cationic agent and ferrous sulphate treated, (b) cationic agent and copper sulphate treated during pre-mordanting process(dyeing condition: temperature $80^{\circ} \mathrm{C}, \mathrm{M}: \mathrm{L}=1: 40$, time $=90 \mathrm{~min}$ ). 

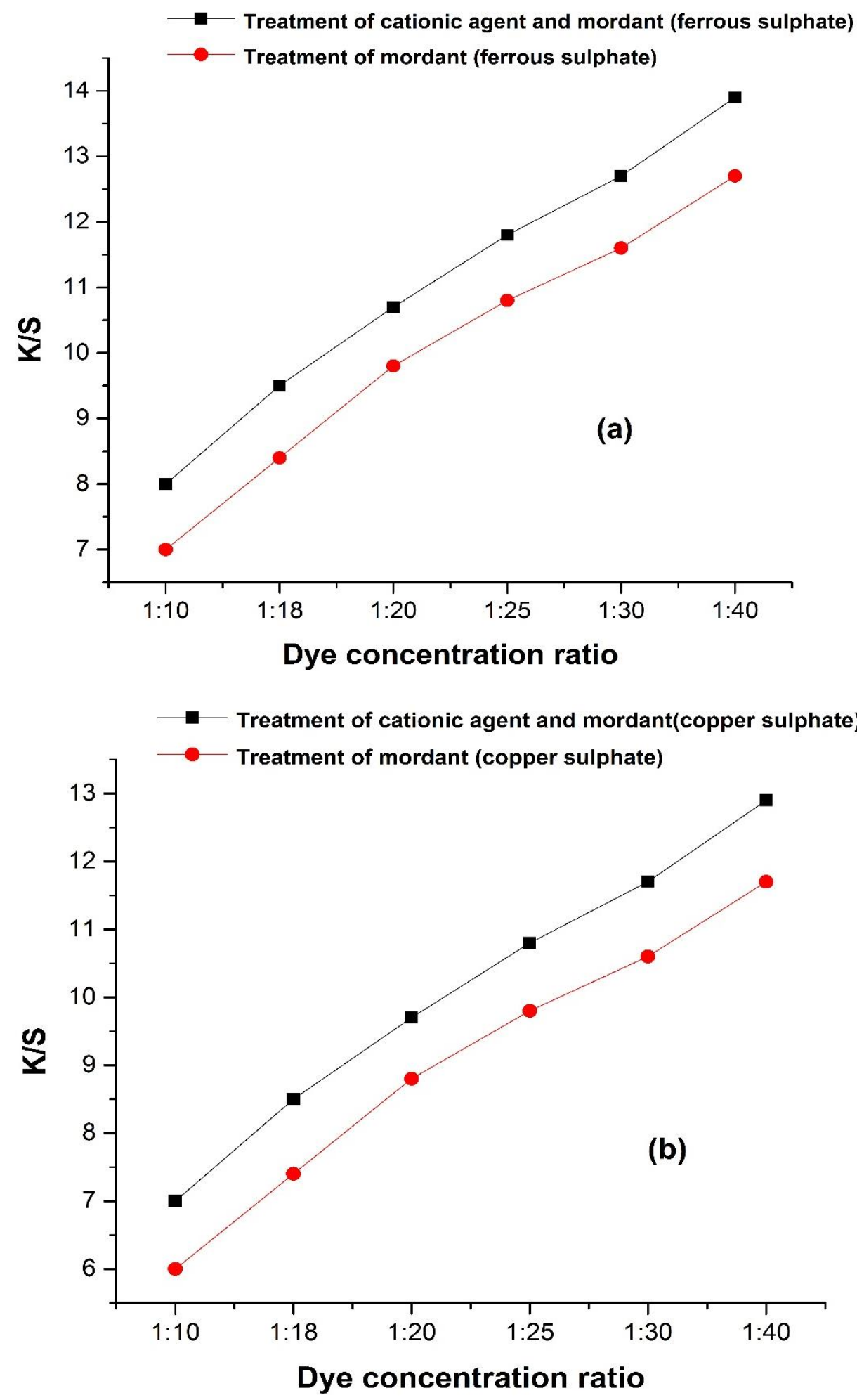

Figure 5: Effect of dyeing dye concentration on the color strength of alginate fiber with (a) cationic agent and ferrous sulphate treated, (b) cationic agent and copper sulphate treated during pre-mordanting process(dyeing condition: temperature $80^{\circ} \mathrm{C}, \mathrm{pH}=7$, time $=90 \mathrm{~min}$ ). 


\subsection{Effect of mordanting and cationisation process on alginate fiber}

Comparative analysis between surface treatment by cationic agent and without any modification of alginate fiber for mordanting process was described at table 3. Brilliant and in depth colorations with good fastness are wanted for packages in a fabric dyeing method. Especially, the use of numerous mordants leads to good sized color difference and in the main effects in a coloration shift towards darkish sunglasses(Jung \& Bae 2014). For increasing the natural attraction between material contribution of natural dye was not meager .In previous study revealed that different metallic mordant did a lot protect the material from devastating effect of environment (Chairat, Bremner, \& Chantrapromma, 2007).

Table 1: Effect of mordanting and cationisation process on alginate fiber

\begin{tabular}{|c|c|c|c|c|c|c|c|c|c|c|}
\hline \multirow[b]{2}{*}{ Mordant } & \multicolumn{5}{|c|}{ Effect of cationisation } & \multicolumn{5}{|c|}{ Effect of Mordant } \\
\hline & $\mathrm{L}^{*}$ & $a^{*}$ & $\mathrm{~b}^{*}$ & $\mathrm{c}^{*}$ & $\mathrm{~K} / \mathrm{S}$ & $\mathrm{L}^{*}$ & $a^{*}$ & $\mathrm{~b}^{*}$ & $\mathrm{c}^{*}$ & $\mathrm{~K} / \mathrm{S}$ \\
\hline $\mathrm{FeSO}_{4}$ & 64.08 & -1.54 & 54.24 & 54.26 & 11.85 & 65.48 & -2.96 & 51.07 & 51.15 & 9.01 \\
\hline $\mathrm{CuSO}_{4}$ & 66.60 & -1.70 & 57.25 & 57.28 & 11.57 & 63.52 & -0.36 & 48.55 & 48.55 & 8.95 \\
\hline $\begin{array}{l}\text { Without } \\
\text { mordant }\end{array}$ & 59.36 & 0.20 & 47.49 & 47.49 & 11.09 & 69.09 & -3.12 & 53.32 & 53.41 & 7.94 \\
\hline
\end{tabular}

*Pre-mordanting

On this take a look at, mordants are used to various the coloration appearances. The utility in a standardized dyeing manner was examined to estimate the ability of dyes from inexperienced rhubarb dye. The technical capability of the extracted materials in regards to the coloration strength and fastness houses of dyeing turned into checked.

The color strength of the alginate fiber dyed with rhubarb dyes was investigated by the data color at Table 1. From different parameters samples we found degree of lightness (L*), degree of depthless $\left(a^{*}\right)$, degree of brightness $\left(b^{*}\right)$, Finally, K/S value was calculated to compare the color strength pre-mordanted ,meta mordanted and post mordanted samples which is given at table . We can see that, that samples which were treated with cationic agent with $\mathrm{FeSO}_{4}$ mordants during pre mordanting method give better results than the mordant treated dyed fiber. From the above result table, it has been found that cationised alginate fiber dyeing with $\mathrm{FeS}_{4}$ mordant gave the $\mathrm{k} / \mathrm{s}$ value 11.85 . On the other hand, alginate fiber dyeing with $\mathrm{FeS}_{4}$ mordant gave the $\mathrm{k} / \mathrm{s}$ value 09.01. Moreover cationised alginate fiber dyeing with $\mathrm{CuSO}_{4}$ mordant gave the $\mathrm{k} / \mathrm{s}$ value 11.57. On the other hand, alginate fiber dyeing with $\mathrm{CuSO}_{4}$ mordant gave the $\mathrm{k} / \mathrm{s}$ value 8.95. Moreover cationised alginate fiber dyeing without any mordant gave the $\mathrm{k} / \mathrm{s}$ value 11.09.Furthermore cationised alginate fiber when dyeing with mordant improves the color strength value of sample when compared to alginate fiber when dyeing with mordant .The physiochemical reaction behind this tremendous change was explained by jothi $\mathrm{D}$ who look over that the ionization of the hydroxyl groups and alkyl halide in the dye molecules under the alkaline condition of the standard detergent solution not only responsible for dramatic color change but also the decomposition of the dye itself, resulting in a colorless or a differentially 
colored compound attributed to dramatic color change (Jothi 2008). There was an inverse relationship existing between natural dye and water soluble properties.

\subsection{SEM analysis}

In case of rhubarb dye SEM shows the surface morphology of the dyed alginate fiber in $3000 \mathrm{X}$ magnifications. From the above SEM micrograph of the Figure 6, it shows that the micrograph at $3000 \mathrm{X}$ in Figure 6 (a) is more smooth in surface than micrograph at 3000X in Figure 6 (b) and Figure 6 (c).Due to cationisation of alginate fiber after dyeing, there is significant change in the surface.

Surface of alginate fiber at $3000 \mathrm{X}$ as shown in Figure 6 (a).For the consequence dye particle evenly distribute to the surface of alginate fiber as shown in figure Figure 6 (a).On the other hand surface of mordanted alginate fiber after dyeing is not smooth as compared to cationised alginate fiber as shown in Figure 6 (b).
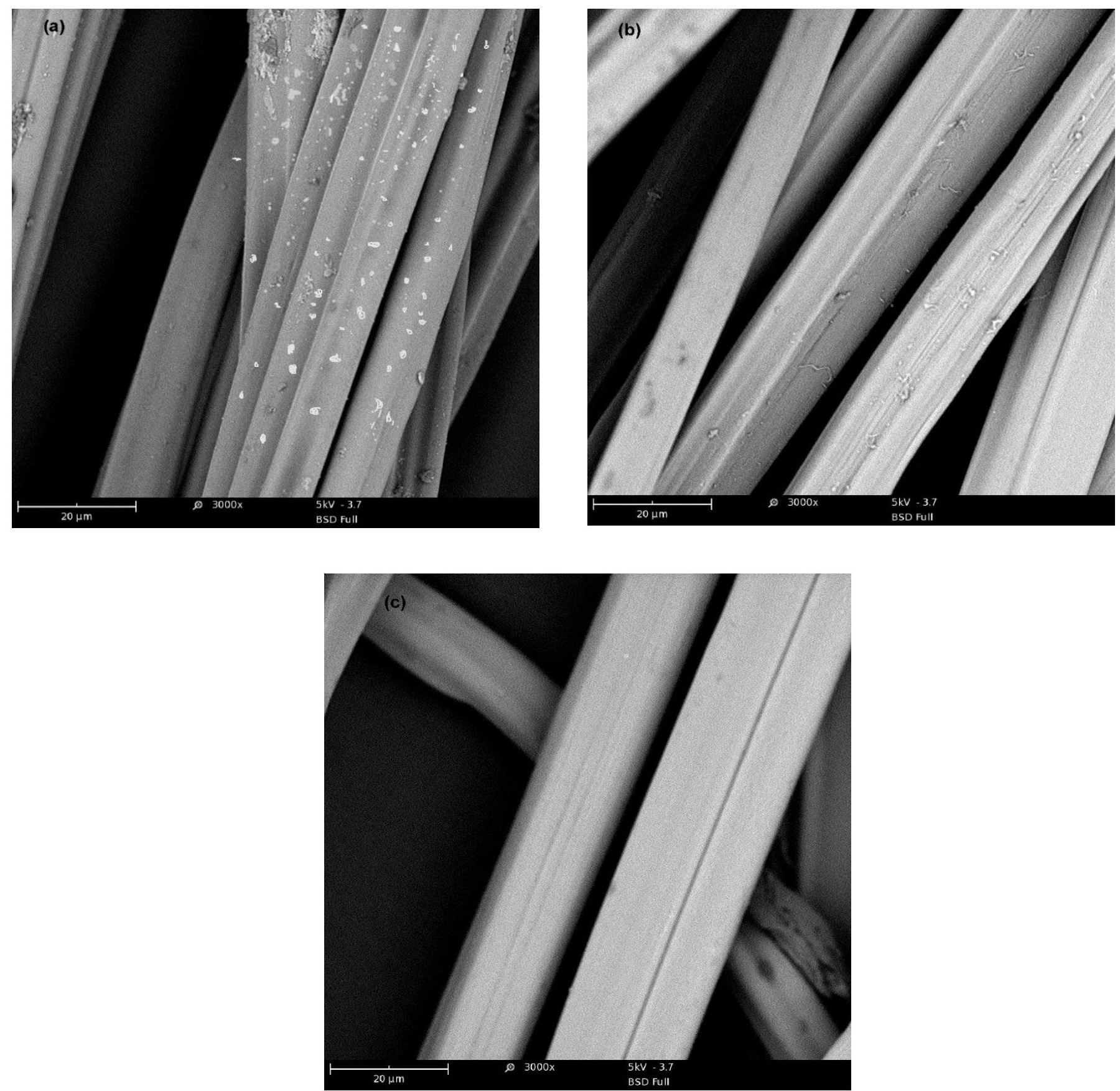
Figure 6 : SEM Micrograph of (a) cationised and mordanted alginate fiber, (b) mordanted alginate fiber and (c) raw alginate fiber dyed with rhubarb dye.

\subsection{Breaking strength}

Table 2: Breaking strength of alginate fiber

\begin{tabular}{llll}
\hline & & Effect & \multicolumn{2}{c}{ of } & Effect of mordant \\
Mordant & Alginate fibers (cN/dtex) & $\begin{array}{l}\text { Cationisation with with alginate alginate } \\
\text { dyed fiber(cN/dtex) } \\
\text { fiber(cN/dtex) }\end{array}$ \\
\hline $\mathrm{FeSO}_{4}$ & 2.35 & 2.02 & 2.02 \\
$\mathrm{CuSO}_{4}$ & 2.35 & 2.05 & 2.05 \\
Without mordant & 2.35 & 2.01 & 2.01 \\
\hline
\end{tabular}

*Pre-mordanting

Breaking strength of dyed alginate fiber was analyzed on the basis two methods such as cationisation and mordanting method which was shown at Error! Reference source not found.. It was seen from the table that generally alginate fiber breaking strength was respectively 2.35 $\mathrm{cN} /$ dtex, $2.35 \mathrm{cN} /$ dtex, $2.35 \mathrm{cN} /$ dtex when mordanting was done by using metal mordant respectively ferrous sulphate, copper sulphate without any mordant. When alginate fiber treated with cationic agent and mordant before

Dyeing it was obvious that breaking strength of alginate was decrease as compared to original alginate fiber.The reason for this scenario was due to the effect of natural dye that destroyed the high packing density of fiber. However the dyeing parameter of alginate fiber had no tremendous influence on the mechanical property of alginate fiber

\subsection{Wash fastness}

Table 3: Wash fastness of alginate fiber

\begin{tabular}{|c|c|c|c|c|}
\hline \multirow[b]{2}{*}{ Mordant } & \multicolumn{2}{|c|}{ Effect of cationisation } & \multicolumn{2}{|c|}{ Effect of mordanting } \\
\hline & Color Stain & $\begin{array}{l}\text { Color } \\
\text { change }\end{array}$ & Color Stain & Color change \\
\hline $\mathrm{FeSO}_{4}$ & $3-4$ & 4 & $2-3$ & 4 \\
\hline $\mathrm{CuSO}_{4}$ & 3 & 4 & 3 & 4 \\
\hline $\begin{array}{l}\text { Without } \\
\text { mordant }\end{array}$ & $3-4$ & 4 & 3 & 4 \\
\hline
\end{tabular}

*Pre-mordanting

In this experiment, we done washing fastness test on rhubarb dyed alginate fiber dyeing with different mordant that was shown at Error! Reference source not found.. Different samples color fastness to wash was done with multi fiber fabrics and the samples staining were invested on cotton part of the multi-fiber fabric after washing. In case of cotton part, we have found that, cationized alginate fiber dyed with rhubarb dyes solution using different mordant in dyeing 
machine gave the best fastness grade as compared to pre-mordanted dyed fiber. In case of solution of cationized alginate fiber dyed with rhubarb dyes solution using ferrous sulphate mordant in dyeing machine gave the best (3-4) fastness grade as compared to dyed fiber with ferrous sulphate mordant which are shown in Table 3. Cationized alginate fiber dyed with rhubarb dyes solution using copper sulphate mordant in dyeing machine gave the higher (3) fastness grade as like to dyed fiber with copper sulphate mordant. Moreover Cationized alginate fiber dyed with rhubarb dyes solution without any mordant in dyeing machine gave the similar (3-4) fastness grade when compared to dyed fiber without metal mordant through the assessment of grey scale.

\section{Conclusion}

Alginate fiber was modified by using cationising agent and mordant during dyeing with rhubarb dye that is decent method. The alginate fiber was dyed by following two approach at one approach cationised and mordanted alginate fiber dyed with rhubarb dye at temperature $80^{\circ} \mathrm{C}$, form $90 \mathrm{~min}$ at $\mathrm{pH} 7$ by maintain liquor ration 1:40. On the other approach mordanted alginate fiber dyed with rhubarb dye at temperature $80^{\circ} \mathrm{C}$, form $90 \mathrm{~min}$ at $\mathrm{pH} 7$ by maintain liquor ration 1:40. The method of cationisation improves the dyeing performance of alginate fiber with rhubarb dye. The test results like Fourier Transform Infrared Spectroscopy (FTIR) of the extracted powder form and SEM of alginate fiber was good. After dyeing, different tests of the dyed sample was done like color strength test $(\mathrm{k} / \mathrm{s})$, color fastness to wash to check the fastness properties of alginate fiber. According to the test results of the different dyed sample, washing fastness of the all pre-mordanted and cationised dyed fiber was good as compared to postmordanted dyed fiber. Color yield value for dyes solution used in dyeing for cationised alginate fiber was evaluated by $(\mathrm{k} / \mathrm{s})$ value that was good for pre-mordanted fiber. Finally, cationising method of alginate fiber with Rhubarb dyes is comparatively good in comparing with different test results rather than the other traditional methods. Its fastness property was good; process is simple, high colour yield than other conventional methods and can be used for the further commercial use.

\section{References}

Badwan, AA, Abumalooh, A, Sallam, E, Abukalaf, A, \& Jawan, O. (1985). A sustained release drug delivery system using calcium alginate beads. Drug Development and Industrial Pharmacy, 11(2-3), 239-256.

Burrows, T, \& Welch, MJ. (1983). The development and use of alginate fibres in nonwovens for medial end-users. Paper presented at the Nonwoven conference papers. Manchester, UK7 UMIST.

Chairat , M, Bremner, J B, \& Chantrapromma, K. (2007). Dyeing of cotton and silk yarn with the extracted dye from the fruit hulls of mangosteen, Garcinia mangostana linn. Fibers and Polymers, 8, 613-619. 
Dey, Kamol, Khan, Ruhul A, \& Chowdhury, AM Sarwaruddin. (2011). Fabrication and mechanical characterization of calcium alginate fiber-reinforced polyvinyl alcohol based composites. Polymer-Plastics Technology and Engineering, 50(7), 698-704.

Gacesa, Peter. (1988). Alginates. Carbohydrate polymers, 8(3), 161-182.

Grant, Gregor T, Morris, Edwin R, Rees, David A, Smith, Peter JC, \& Thom, David. (1973). Biological interactions between polysaccharides and divalent cations: the egg-box model. FEBS letters, 32(1), 195-198.

Hong-bin, LIU. (2005). WANG Yong-yue 1, XIAO Chang-fa 1 (1. School of Materials Science and Chemical Engineering, Tianjin Polytechnic University, Tianjin 300160, China; 2. Department of Science and Technology Development, China Petroleum \& Chemical Corporation, Beijing 100029, China); Research on preparation and properties of salt alginate fiber [J]. Journal of Tianjin Institute of Textile Science and Technology, 3.

Jothi , D. (2008). Extraction of natural dyes from African marigold flower (Tageteserecta L.) for textile coloration. AUTEX Res. J, 8, 49-53.

Jung, Y S , \& Bae , D G. (2014). Natural dyeing with black cowpea seed coat. I. Dyeing properties of cotton and silk fabrics. Fibers and Polymers, 15, 138-144.

King, AH. (1983). Brown seaweed extracts (alginates). Food hydrocolloids, 2, 115-188.

LIU, Rui-ning, \& GAO, Guang-dong. (2010). Cotton dyeing with natural gardenia yellow dye [J]. Dyeing \& Finishing, 5, 008.

Luo, Yongxiang, Lode, Anja, \& Gelinsky, Michael. (2013). Direct Plotting of ThreeDimensional Hollow Fiber Scaffolds Based on Concentrated Alginate Pastes for Tissue Engineering. Advanced healthcare materials, 2(6), 777-783.

Morris, Edwin R, Rees, David A, Thom, David, \& Boyd, Jonathan. (1978). Chiroptical and stoichiometric evidence of a specific, primary dimerisation process in alginate gelation. Carbohydrate research, 66(1), 145-154.

Ott, CM, \& Day, DF. (1995). Bacterial alginate: an alternative industrial polymer. Trends in polymer science, 3(12), 402-406.

Properties, ASTM Subcommittee D20. 10 on Mechanical. (1996). Standard test method for tensile properties of plastics.

Qin, Y, Agboh, Ch, Wang, X, \& Gilding, DK. (1996). Alginate fibers. Chemical Fibers International, 46(4), 273.

Siviello, Ciro. (2016). Studio delle dinamiche dei gel fisici: gel ionici a base di alginato.

Skjåk-Bræk, Gudmund. (1992). Alginates: biosyntheses and some structure-function relationships relevant to biomedical and biotechnological applications. Biochemical Society Transactions, 20(1), 27-33.

Skoda, Frantisek, Astier, Marcel P, Pajonk, Gérard M, \& Primet, Michel. (1994). Surface characterization of palladium-copper bimetallic catalysts by FTIR spectroscopy and test reactions. Catalysis letters, 29(1), 159-168.

Thomas, Adrian, Gilson, Christopher D, \& Ahmed, Tanveer. (1995). Gelling of alginate fibres. Journal of Chemical Technology and Biotechnology, 64(1), 73-79.

Vankar, Padma S, Shanker, Rakhi, \& Verma, Avani. (2007). Enzymatic natural dyeing of cotton and silk fabrics without metal mordants. Journal of Cleaner Production, 15(15), 14411450.

Wang, Ping, Tawiah, Benjamin, Tian, Anli, Wang, Chunxia, Zhang, Liping, \& Fu, Shaohai. (2015). Properties of alginate fiber spun-dyed with fluorescent pigment dispersion. Carbohydrate polymers, 118, 143-149. 
Yimin, Qin. (2003). The Application of Alginate Fiber in the Medical Dressing [J]. Synthetic Fiber in China, 4, 002.

ZHAN, Yi-zhen, ZHU, Ping, ZHANG, Jian-bo, \& GUO, Xiao-qing. (2006). The properties and application of alginate fiber [J]. Textile Auxiliaries, 6, 002.

Zhu, Ping, Zhang, Chuanjie, Sui, Shuying, \& Wang, Huaifang. (2009). Preparation, structure and properties of high strength alginate fiber. Research Journal of Textile and Apparel, 13(4), $1-8$. 